\title{
DEVELOPMENT OF A RADIAL BASIS FUNCTION NETWORK TO ESTIMATE THE HEAD GENERATED BY ELECTRICAL SUBMERSIBLE PUMPS ON GASEOUS PETROLEUM FLUIDS
}

\author{
Morteza Mohammadzaher ${ }^{1}$, Mojataba Ghodsi ${ }^{1}$ and Abdullah AlQallaf ${ }^{2}$ \\ ${ }^{1}$ Department of Mechanical and Industrial Engineering, \\ Sultan Qaboos University, Muscat, Oman \\ ${ }^{2}$ Department of Electrical Engineering, Kuwait University \\ Kuwait City, Kuwait
}

\begin{abstract}
This paper proposes radial basis function network (RBFN) models to estimate the head of gaseous petroleum fluids (GPFs) in electrical submersible pumps (ESPs) as an alternative to widely used empirical models. Both exact and efficient RBFN modelling approaches were employed. RBFN modelling essentially tend to minimise the modelling error, the discrepancy of estimated and real outputs within the modelling data. This may lead to overfitting and lack of model generality for operating conditions not reflected in modelling data. This critical matter was addressed in RBFN design process, and highly accurate RBFNs were developed and cross validated.
\end{abstract}

\section{KEYWORDS}

Electrical Submersible Pump(ESP), Radial Basis Function Network (RBFN), Model, Petroleum, Gaseous, Head Estimation

\section{INTRODUCTION}

ESPs are widely used to lift large volume of fluid from downhole at different well conditions [1, 2]. As a vital task, size of these pumps should be chosen correctly as over- or under-sizing leads to premature equipment failure or low petroleum fluid recovery. In order to facilitate size selection, manufacturers normally provide curves depicting generated head versus liquid volumetric flow rate for each ESP size, the size of ESPs is selected based on manufacturer curves.

However, the aforementioned curves are not valid for gaseous fluids; where, ESPs are utilised to pump two-phase fluid with high gas content [1].The solution is to develop models to estimate the generated head by ESPs on GPFs. This paper focuses on head estimating models and exclude other types of models developed for GFPs in ESPs, e.g. the ones which estimate surging or stability border [3], gas bubble size [4] or in-situ gas volume fraction [5]. 
Head-estimating models have been developed and investigated for decades using analytical, numerical and empirical approaches [6]. Analytical models have been derived based on mass and momentum balances [7, 8]. Use of unrealistic assumptions and oversimplification of complex physics of two-phase fluids have weaken the reliability of analytical models. Numerical models are not trusted too as they are normally formulated based on one-dimensional two-fluid conservations of mass and momentum along streamlines and require the prediction of surging initiation in ESPs [9]. On the contrary, empirical models of GFPs in ESPs are widely trusted and used in practice $[10,11]$.

\section{EMPIRICAL MODELS}

In this section, homogenous model and a number of empirical head-estimating models of GPFs in ESPs are briefly introduced. The parameters of the presented empirical models have been identified using the data collected from experiments on diesel fuel/carbon dioxide mixtures, reported in [12]. Aforementioned mixtures are similar to petroleum fluids [13]. A number of other empirical models have been also reported in the literature in which their parameters have been identified based on the data of experiments on air/water mixtures [14-16]. These models have been excluded from this paper due to dissimilarity of the tested fluids and GPFs.

\subsection{Model 1}

The hoariest model is the homogenous model. This model is in fact a brief analytical model rather than an empirical model, based on oversimplification of two-phase physics of GPFs. Homogenous model receives an input from the curve provided by the manufacturer: the generated head by ESP if pure liquid was pumped instead of GPF (Hl ) [17]. This head is modified with assumption that the fluid motion is homogenous i.e. liquid and gas have equal speeds:

$$
\hat{H}_{m}=\left((1-\alpha) \rho_{l}+\alpha \rho_{g}\right) H_{l},
$$

where $\rho, H$ and indices $l, g$ and $m$ stand for density, head, liquid, gas, and mixture, respectively. $\alpha$ is gas void fraction. ${ }^{\wedge}$ shows the head is estimated (not experimentally measured).

\subsection{Model 2}

The second model was developed by Turpin et al in 1986 [18]:

$$
\hat{H}_{m}=H_{l} \exp \left(346430\left(\frac{q_{g}}{p_{\text {in }} q_{l}}\right)^{2}-410\left(\frac{q_{g}}{p_{\text {in }} q_{l}}\right)\right) \text {, }
$$

where $q_{l}$ and $q_{g}$ are liquid and gas volumetric flow rates in gallons per minutes $(\mathrm{gpm}), p_{\text {in }}$ is intake pressure in $p s i$.

\subsection{Model 3}

This model was proposed by Sachdeva et al, in 1992 [19]:

$$
\hat{H}_{m}=\frac{K_{1}}{\rho_{m} g} p_{i n}^{{ }^{E}} \alpha^{E 2} q_{l}^{E 3} .
$$

where $g$ stands for gravity acceleration. The values of $E_{1}, E_{2}$ and $E_{3}$ and $K_{1}$ are listed in [10] for multiple stages of electrical submersible pumps. As an example, for 8 stages of I-42B radial ESP, $K_{1}=1.1545620, E_{1}=0.943308, E_{2}=-1.175596$ and $E_{3}=-1.300093$. Similar to Model 2, (3) is 
convertible to a linear equation through taking algorithm. Parameters of a linear equation can be often identified straightforwardly using least square of error algorithm [20]

\subsection{Model 4}

This model was presented by Zhou and Sachdeva in 2010 [10]:

$$
\hat{H}_{m}=H_{\text {max }} K_{2}\left(C p_{i n}\right)^{\alpha E_{4}}(1-\alpha)^{E_{5}}\left(1-\frac{q_{m}}{q_{\max }}\right)^{E_{6}},
$$

where $K_{2}$ is a unit-less coefficient, $C$ is pressure unit factor, e.g. 1, 1000 or 0.145 for psi, ksi or $k P a . H_{\max }$ and $q_{\max }$ are nominal maximum head and flow rate of the ESP; $q_{m}$ is mixture or GPF flow rate where $q_{m}=q_{l}+q_{g}=q_{g} / \alpha$.

Mathematical structure of this model seems more meaningful than Model 3; as if gas void fraction and flow rate equal zero, estimated head is definitely $H_{\max }$. According to [10], for 8 stages of I-42B radial ESP, $K_{2}=1.971988, E_{4}=1.987838, E_{5}=9.659664$ and $E_{6}=0.905908$.

\subsection{Summary and Limits of Empirical Models}

All presented models have three input variables amongst $p_{i n}, \rho_{l}, \rho_{g}, q_{l}, q_{g}, q_{m}$ or $\alpha$. Two other potential input variables, pump rotational speed and temperature are missing in all empirical models of GPFs in ESPs. In fact, the parameters of the presented models have been identified based on the data collected at a fixed rotational speed of $3500 \mathrm{rpm}$; thus, the models are valid merely at this speed. The estimated head can be adapted for other rotational speeds using 'affinity laws' detailed in $[2,10]$.

\section{MODEL DEVELOPMENT}

In this research, a radial basis function network (RBFN) was developed to estimate the head of mixtures of carbon dioxide/ diesel fuel pumped by eight stages of an I-42B radial ESP. The reason to choose an RBFN as the head-estimating model is the fact that RBFNs are universal approximators with significant mathematically proven modelling capabilities [21]. Inspired by existing empirical models, a single output of $H_{m}$ and triple inputs of $p_{i n}, q_{m}$ and $\alpha$ were opted for the RBFN model. The same experimental data, which were used to identify the parameters of empirical models 1-4, were utilised to develop and test the RBFN. Thus, the proposed model and presented empirical models are comparable. The experimental data cover a wide range of gas void fractions [0 0.5], intake pressures [50 to 400] psi and heads [1 55] ft. 110 sets of input/output data are available, in this research, 93 were used for modelling and 17 sets for testing the RBFN.

An RBFN has two layers, the first layer receives inputs array $(\mathbf{U})$ and produces the 'layer output' $(\mathbf{O})$.The second layer receives $\mathbf{O}$ and produces the 'network output' $(\mathbf{Y})$. In this problem, with three inputs and one output, the input and output arrays are $\mathbf{U}_{3 \times Q}$ and $\mathbf{Y}_{1 \times Q} . Q$ is the number of data sets which are fed to the RBFN at once. For instance, if the inputs of the modelling data are fed into the model altogether, then $Q=93$.

The first layer has an array of weights $\left(\mathbf{W}_{R \times 3}\right)$ and a scalar namely Spread $(S)$. The components of layer output, $\mathbf{O}_{R \times Q}$, are calculated as following: 


$$
\mathbf{O}_{i k}=\exp \left(-(\underbrace{2}_{\begin{array}{c}
S \\
\sum_{j=1}^{3}\left(\mathbf{W}_{i j}-\mathbf{U}_{j k}\right)^{2} \\
\text { and weighte artant input }
\end{array}})^{2}\right) .
$$

The second layer has an array of weights $\left(\mathbf{X}_{1 \times R}\right)$ as well as an array of biases $\left(\mathbf{B}_{1 \times Q}\right)$. The output array is calculated as following:

$\mathbf{Y}_{1 \times Q}=\mathbf{X}_{1 \times R} \times \mathbf{O}_{R \times Q}+\mathbf{B}_{1 \times Q}$,

Combination of (5) and (6) is the structure of the RBFN; the next task is to identify its unknown parameters $R, \mathbf{W}, S, \mathbf{X}$ and $\mathbf{B}$ using the modelling data including input and output vectors of $\mathbf{U}_{3 \times 93}$ and $\mathbf{Y}_{1 \times 93}$.

From (5), it is clear that the range of $\mathbf{O}$ components is [0 1]; also, if $i^{\text {th }}$ row of $\mathbf{W}$ and $k^{\text {th }}$ column of $\mathbf{U}$ are identical, $\mathbf{O}_{i k}$ will be at its maximum, 1; or simply, maximum values of $\mathbf{O}$ components happen if the rows of $\mathbf{W}$ are same as the columns of $\mathbf{U}$. From (6), it can be seen larger components of $\mathbf{O}$ are more influential on the network output. As a result, in order to maximise the effect of the modelling data on parameter selection, it has been suggested to set $\mathbf{W}=\mathbf{U}^{\mathbf{T}}$, consequently $R=Q$. Then, $\mathbf{O}$ can be calculated with $\mathbf{U}_{3 \times 93}$ of the modelling data and an $S$ according to (5).

$\mathbf{B}$ and $\mathbf{X}$ can also be found from linear equation of (6). In practice, Eq. (6) in the form of (7) was solved to find $\mathbf{B}$ and $\mathbf{X}$ :

$$
\mathbf{Y}_{1 \times 93}=\left[\begin{array}{ll}
\mathbf{X} & \mathbf{B}
\end{array}\right]_{1 \times 186}\left[\begin{array}{l}
\mathbf{O} \\
\mathbf{I}
\end{array}\right]_{186 \times 93},
$$

where I is a unique matrix with size of $93 \times 93$.

By this point, it has been shown how to find all unknowns of (5) and (6) except for $S$. The developed model is called an 'exact' RBFN. Such a model evidently provides exact estimation for the modelling data; however, a serious concern about exact RBFNs is inaccuracy of estimation outside the operating points where the modelling data have been collected from. A large spread $(S)$ ( $S>>1$ in (5)) can smoothen the model output and generalise the network [22].

Here is a pseudo-algorithm of exact RBFN modelling (to find $R, \mathbf{W}, \mathbf{X}, \mathbf{B}$ and $S$ using the input and out arrays of the modelling data, $\mathbf{U}_{3 \times 93}$ and $\mathbf{Y}_{1 \times 93}$ )

1. Set $\mathbf{W}_{93 \times 3}=\mathbf{U}_{93 \times 3}^{\mathbf{T}}$

2. Choose a large $S$ to generalise the developed RBFN

3. Calculate $\mathbf{O}_{93 \times 93}$ from (5) with $\mathbf{U}_{3 \times 93}$ (from the modelling data), $\mathbf{W}_{93 \times 3}$ and $S$ defined at steps 1 and 2 .

4. Form (7) with $\mathbf{Y}_{1 \times 93}$ (from the modelling data) and $\mathbf{O}$ calculated at step 3.

5. Solve (7) to find $\mathbf{X}_{1 \times 93}$ and $\mathbf{B}_{1 \times 93}$

A straightforward non-iterative parameter identification algorithm is an advantage of exact RBFNs; however, this method creates models with too many parameters: 466 in this research. While, only 93 modelling data sets, in total 372 pieces of input/output data, are available. Excessive number of parameters and focus of the algorithm on exact fitting of the model to the modelling data increases the risk of 'overfitting' or lack of generality (see [23, 24]). Spread as the only tool to generalise the algorithm has shown to be insufficient for this purpose [23]. 
An alternative is to use efficient RBFNs which may have much fewer parameters than exact RBFNs. In exact RBFNs, all columns of input data, $\mathbf{U}$, are transposed and used as the rows of weight array, W. In efficient RBFNs, some columns of $\mathbf{U}$ are selected and transposed to form $\mathbf{W}$. Thus, $\mathbf{W}$ array is smaller. In order to select $\mathbf{U}$ columns to be used as $\mathbf{W}$ rows, first, every single column is transposed and tried as a single-row $\mathbf{W}$. The column of $\mathbf{U}$ which leads to the smallest modelling error (see the second appendix of [25] about the modelling error) is selected, transposed and used as the first row of $\mathbf{W}$. At the next iteration, another column of $\mathbf{U}$ in which the merger of its transpose to $\mathbf{W}$ leads to the largest drop in the modelling error is chosen and added to $\mathbf{W}$. This continues till the number of $\mathbf{W}$ rows $(R)$ reaches its pre-defined maximum $\left(R_{\max }\right)$ or the modelling error reaches its predefined target $\left(E_{\mathrm{t}}\right)$. It should be noted that if a too small modelling error (e.g. 0) is targeted, overfitting is more likely to happen.

Here is a pseudo-algorithm of efficient RBFN modelling:

1. $\mathbf{W}=$ null, $\mathbf{U}_{\text {rem }}=\mathbf{U}, \mathbf{U}_{\text {opt }}=$ null, $E=1000$ (a large number)

2. Choose a large $S$ to generalise the developed RBFN

3. Choose $R_{\max }$ and target modelling error, $E_{\mathrm{t}}$

4. Set $R=1$

5. Set $k=1$

6. Add transpose of $k^{\text {th }}$ column of $\mathbf{U}_{\text {rem }}$ as the $R^{\text {th }}$ row of $\mathbf{W}$

7. Calculate $\mathbf{O}$ from (5) with $\mathbf{U}_{3 \times 93}$ (from the modelling data), $\mathbf{W}_{R \times 3}$ and $S$ defined at steps 6 and 2.

8. Solve $\mathbf{Y}_{1 \times 93}=\left[\begin{array}{ll}\mathbf{X} & \mathbf{B}\end{array}\right]_{1 \times(R+93)}\left[\begin{array}{l}\mathbf{O} \\ \mathbf{I}\end{array}\right]_{(R+93) \times 93}$ to find $\mathbf{X}_{1 \times R}$ and $\mathbf{B}_{1 \times R}(\mathbf{Y}$ and $\mathbf{O}$ are available from the modelling data and step 7)

9. Find the Modelling Error (ME) from comparison of $\mathbf{Y}_{\text {model }}$ (calculated from (5) and (6)) and $\mathbf{Y}$

10. if $M E<E$, then $E=M E$ and $\mathbf{U}_{\mathrm{opt}}=\mathbf{U}_{k}$

11. $k=k+1$

12. if $k \leq 93-R$ then go to 6

13. Remove $\mathbf{U}_{\text {opt }}$ from $\mathbf{U}_{\text {rem }}$

14. $R=R+1$

15. if $R \leq R_{\max }$ and $E>E_{\mathrm{t}}$ then go to 5

\section{RESULTS AND DISCUSSION}

Both exact and efficient RBFN modelling methods were employed to develop models for GPFs pumped by eight stages of an I-42B radial ESP, using 93 sets of experimental data as detailed in the previous section. Accuracy of the models were tested with 17 data sets not used for modelling, 'test data'. Test data include an input array of ${ }^{\mathrm{T}} \mathbf{U}_{3 \times 17}$ and an output vector of ${ }^{\mathrm{T}} \mathbf{Y}_{17 \times 1}$. Upper left index of T refers to 'test'. The estimated outputs of a model with inputs of ${ }^{\mathrm{T}} \mathbf{U}_{3 \times 17}$ is named ${ }^{\mathrm{T}} \hat{\mathbf{Y}}_{17 \times 1}$. The 'test error' or $T E$, as defined in (8), was used to assess the accuracy of models. 
$T E=\frac{\sum_{l=1}^{17}\left|{ }^{\mathrm{T}} \mathbf{Y}_{l}-{ }^{\mathrm{T}} \hat{\mathbf{Y}}_{l}\right|}{17}$,

A model should lead to an acceptably low $T E$ to be cross-validated [25, 26]. Exact RBFN, with 466 parameters, provides a fairly good $T E, 2.7683 \mathrm{ft}$, at a very high value of spread, $S=125$. At lower values of $S$, the modelling error may be misleadingly small. For instance, at $S=1$, if the modelling data are used in (8), the resultant error is $0.0645 \mathrm{ft}$ where the generalisation [26] or test error is $44.8621 \mathrm{ft}$, an obvious case of overfitting to the modelling data.

The efficient RBFN, however, provides better results with fewer parameters and a lower spread. A spread of 20 and target modelling error $\left(E_{\mathrm{t}}\right)$ of $1.2 \mathrm{ft}$ result in an efficient RBFN with $R=74$, total number of parameters of 390 and an excellent test error of $1.8648 \mathrm{ft}$, i.e. $3.45 \%$ of head range. As a result, this model is cross-validated too.

Table 1 compares the test error (TE) and number of parameters in empirical models 1-4 (M1-M4) and developed exact and efficient RBFNs.

Table 1. Test error $(T E)$ in $f t$ and total number of parameters for different models

\begin{tabular}{|l|c|c|c|c|c|c|}
\hline & M1 & M2 & M3 & M4 & Exact RBFN & Efficient RBFN \\
\hline Test Error & 8.85 & 7.36 & 12.13 & 5.16 & 2.77 & 1.87 \\
\hline Number of Parameters & 1 & 3 & 4 & 5 & 466 & 390 \\
\hline
\end{tabular}

Figures 1-3 compare estimated heads of different models with the real head at three different operating areas. In this paper, an operating area is the collection of operations at a fixed intake pressure and a fixed gas void ratio, e.g. $P_{\mathrm{in}}=100 \mathrm{psi}$ and $\alpha=0.2$. Table 2 shows the mean of absolute estimation error for different operating areas. The results presented in Table 2 and Figs.1-3 have been calculated for the entire available experimental data in each operating area, not only the tests data.

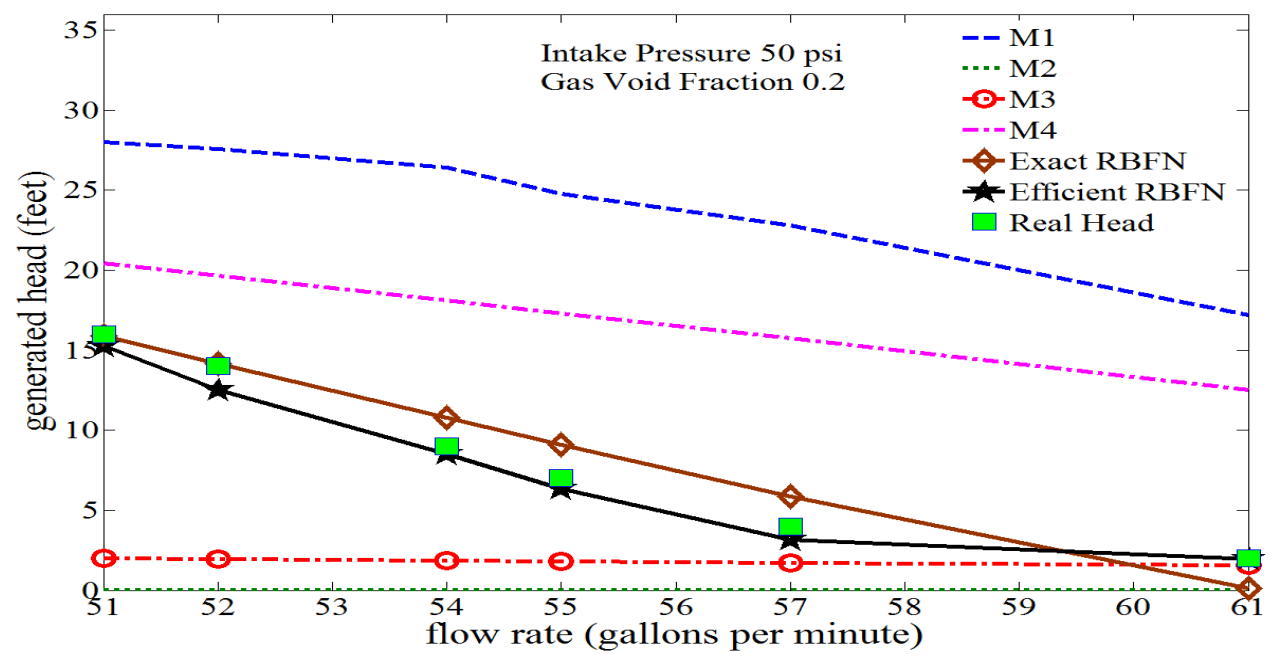

Figure 1. Real and estimated head (by six models) for a mixture of carbon dioxide and diesel fuel pumped by eight stages of an I-42B radial ESP; intake pressure is 50 psi and gas void fraction is 0.2 


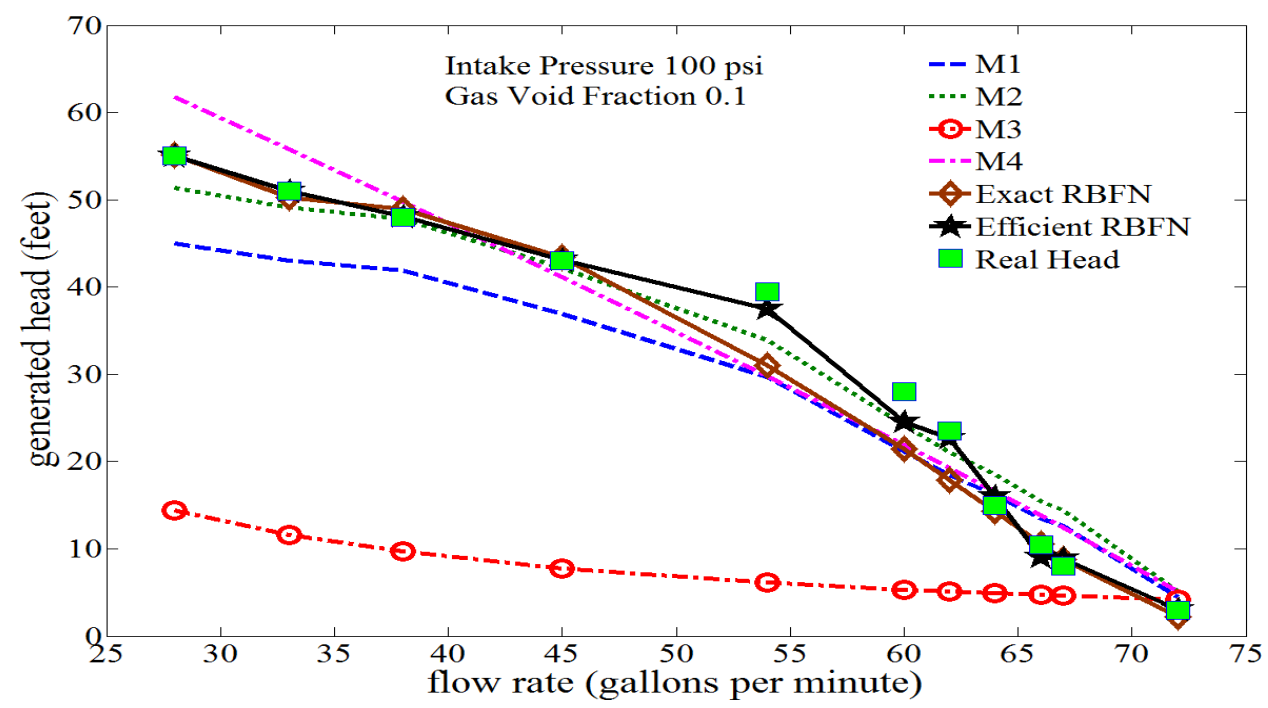

Figure 2. Real and estimated head (by six models) for a mixture of carbon dioxide and diesel fuel pumped by eight stages of an I-42B radial ESP; intake pressure is $100 \mathrm{psi}$ and gas void fraction is 0.1

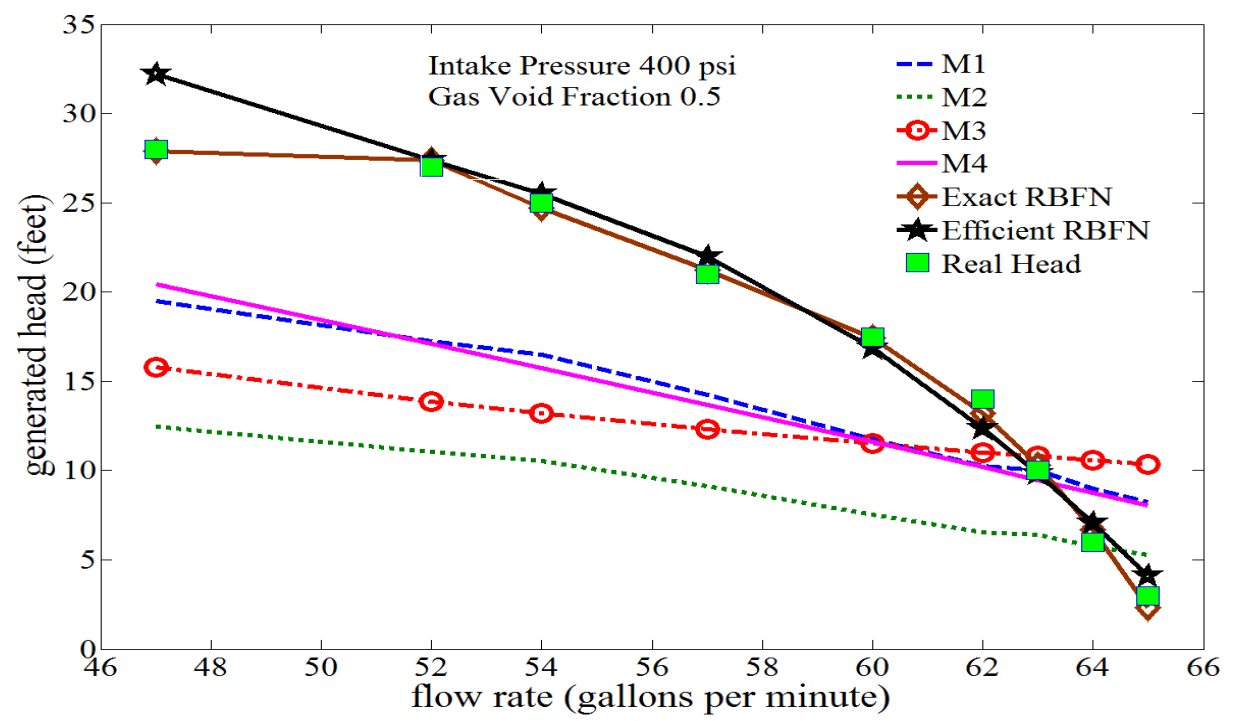

Figure 3. Real and estimated head (by six models) for a mixture of carbon dioxide and diesel fuel pumped by eight stages of an I-42B radial ESP; intake pressure is $400 \mathrm{psi}$ and gas void fraction is 0.5

As to Table 2, in 13 operating areas covered by experiments, the efficient and exact RBFN models outperform all comparable empirical models, M1-M4, in 12 and 11 operating areas respectively. Only in one operating area, Model 3 presents a lower error than RBFNs, at pressure of $50 \mathrm{psi}$ and gas void ratio of 0.4 . In this operating area only 2 sets of experimental data are available. In both Table 2 and Figs. 1-3, it is observed that different empirical models may perform better in specific operating areas; thus, so called critical models [6] have been proposed to define the validity area of some empirical models. As an advantage, RBFN models are valid for the whole operating areas where the modelling and test data have been collected from. 
Table 2. Mean of absolute head estimation error in $f t$ for different models at various operating areas.

\begin{tabular}{|c|l|c|c|c|c|c|c|}
\hline$P_{\text {in }}$ & $\alpha$ & M1 & M2 & M3 & M4 & Exact RBFN & Efficient RBFN \\
\hline 50 & 0.10 & 4.66 & 14.7 & 24.1 & 5.00 & 1.42 & 0.33 \\
\hline 50 & 0.15 & 12.1 & 12.0 & 11.5 & 7.32 & 4.62 & 1.85 \\
\hline 50 & 0.20 & 15.8 & 8.63 & 6.85 & 8.63 & 1.32 & 0.71 \\
\hline 50 & 0.30 & 16.4 & 6.63 & 5.06 & 4.34 & 4.97 & 1.68 \\
\hline 50 & 0.40 & 17.1 & 3.00 & 1.39 & 2.44 & 2.31 & 1.81 \\
\hline 100 & 0.10 & 5.66 & 3.21 & 22.5 & 4.24 & 2.29 & 0.90 \\
\hline 100 & 0.15 & 6.28 & 4.61 & 13.3 & 5.94 & 1.98 & 1.06 \\
\hline 100 & 0.20 & 8.25 & 4.96 & 10.4 & 6.68 & 1.29 & 1.30 \\
\hline 100 & 0.30 & 10.1 & 10.3 & 7.76 & 4.65 & 3.00 & 0.43 \\
\hline 100 & 0.40 & 11.7 & 6.40 & 3.81 & 2.89 & 1.33 & 1.09 \\
\hline 400 & 0.30 & 5.47 & 3.73 & 9.92 & 5.84 & 0.80 & 0.52 \\
\hline 400 & 0.40 & 4.45 & 2.95 & 8.27 & 4.30 & 0.57 & 1.18 \\
\hline 400 & 0.50 & 5.69 & 9.04 & 7.51 & 5.79 & 0.39 & \\
\hline
\end{tabular}

\section{CONCLUSION}

This paper first presented existing empirical models, which estimate the head of gaseous petroleum fluids in ESPs. These include a simple analytical model (the homogenous model) and three empirical models.

Afterwards, the same data used to identify the parameters of aforementioned empirical models were used to develop and test the exact and efficient RBFN models to serve the same function as the empirical models. The developed models outperformed existing models, and the efficient RBFN particularly estimated head highly accurately with a test error equivalent to $3.45 \%$ of head range.

It was also shown that if some popular values were opted for RBFN design factors, e.g. spread of 1 and target modelling error of 0 , the developed model would fail to fulfil cross-validation requirements due to overfitting. In the exact RBFN, a very large spread, 125, was shown to be able to reduce overfitting; this purpose was served with use of a large spread, 20, and a fairly large target modelling error for the efficient RBFN.

\section{REFERENCES}

[1] Y. Bai and Q. Bai, Subsea engineering handbook: Gulf Professional Publishing, 2012.

[2] M. Mohammadzaheri, R. Tafreshi, Z. Khan, M. Franchek, and K. Grigoriadis, "An intelligent approach to optimize multiphase subsea oil fields lifted by electrical submersible pumps," Journal of Computational Science, vol. 15, pp. 50-59, 2016.

[3] L. Barrios and M. G. Prado, "Modeling Two-Phase Flow Inside an Electrical Submersible Pump Stage," Journal of Energy Resources Technology, vol. 133, p. 042902, 2011.

[4] J. Zhu and H.-Q. Zhang, "Numerical Study on Electrical-Submersible-Pump Two-Phase Performance and Bubble-Size Modeling," SPE Production \& Operations, 2017. 
[5] J. Zhu and H.-Q. Zhang, "Mechanistic modeling and numerical simulation of in-situ gas void fraction inside ESP impeller," Journal of Natural Gas Science and Engineering, vol. 36, pp. 144-154, 2016.

[6] M. Mohammadzaheri, R. Tafreshi, Z. Khan, M. Franchek, and K. Grigoriadis, "Modelling of Petroleum Multiphase fluids in ESPs, an Intelliegnt Approach," presented at the Offshore Mediternean Conference, Ravenna, Italy, 2015.

[7] R. Sachdeva, "Two-phase flow through electric submersible pumps," University of Tulsa, 1988.

[8] D. Sun and M. Prado, "Modeling gas-liquid head performance of electrical submersible pumps," Journal of Pressure Vessel Technology, vol. 127, pp. 31-38, 2005.

[9] J. Zhu, X. Guo, F. Liang, and H.-Q. Zhang, "Experimental study and mechanistic modeling of pressure surging in electrical submersible pump," Journal of Natural Gas Science and Engineering, 2017.

[10] D. Zhou and R. Sachdeva, "Simple model of electric submersible pump in gassy well," Journal of Petroleum Science and Engineering, vol. 70, pp. 204-213, 2010.

[11] L. R. Pineda, A. L. Serpa, J. L. Biazussi, and N. A. Sassim, "Operational Control of an Electrical Submersible Pump Working with Gas-Liquid Flow Using Artificial Neural Network " presented at the IASTED International Conference on Intelligent Systems and Control Campinas, Brazil, 2016.

[12] J. F. Lea and J. Bearden, "Effect of gaseous fluids on submersible pump performance," Journal of Petroleum Technology, vol. 34, pp. 922-930, 1982.

[13] M. Ghodsi, N. Hosseinzadeh, A. Özer, H. R. Dizaj, Y. Hojjat, N. G. Varzeghani, et al., "Development of Gasoline Direct Injector using giant magnetostrictive materials," IEEE Transactions on Industry Applications, vol. 53, pp. 521-529, 2017.

[14] M. Romero, "An evaluation of an electrical submersible pumping system for high GOR wells," University of Tulsa, 1999.

[15] R. Cirilo, "Air-water flow through electric submersible pumps," University of Tulsa, Department of Petroleum Engineering, 1998.

[16] J. Duran and M. Prado, "ESP Stages Air-Water Two-Phase Performance-Modeling and Experimental Data," 2003.

[17] A. Qallaf and M. Mohammadzaheri, "A Fuzzy Model to Estimate Head of Gaseous Petroleum Fluids Driven by Electrical Submersible Pumps," presented at the Engineering and Technology, Computer, Basic and Applied Sciences, Sydney, Australia, 2017.

[18] J. L. Turpin, J. F. Lea, and J. L. Bearden, "Gas-Liquid Flow Through Centrifugal Pumps-Correlation of Data," presented at the The Third International Pump Symposium, College Station, Texas, USA, 1986.

[19] R. Sachdeva, D. Doty, and Z. Schmidt, "Performance of Axial Electric Submersible Pumps in a Gassy Well," in SPE Rocky Mountain Regional Meeting, 1992.

[20] M. Mohammadzaheri, L. Chen, A. Ghaffari, and J. Willison, "A combination of linear and nonlinear activation functions in neural networks for modeling a de-superheater," Simulation Modelling Practice and Theory, vol. 17, pp. 398-407, 2009.

[21] M. Mohammadzaheri, L. Chen, and S. Grainger, "A critical review of the most popular types of neuro control," Asian Journal of Control, vol. 16, pp. 1-11, 2012. 
[22] M. Beale, M. Hagan, and H. Demuth. (2017). Neural Network Toolbox ${ }^{\mathrm{TM}}$ User's Guide. Available: https://www.mathworks.com/

[23] G. C. Cawley and N. L. Talbot, "On over-fitting in model selection and subsequent selection bias in performance evaluation," Journal of Machine Learning Research, vol. 11, pp. 2079-2107, 2010.

[24] M. Mohammadzaheri, A. Mirsepahi, O. Asef-afshar, and H. Koohi, "Neuro-fuzzy modeling of superheating system of a steam power plant," Applied Math. Sci, vol. 1, pp. 2091-2099, 2007.

[25] M. Mohammadzaheri, A. Firoozfar, D. Mehrabi, and M. Emadi, "A Virtual Temperature Sensor for an Infrared Dryer," presented at the 9th IEEE-GCC Conference and Exhibition, Manama, Bahrain, 2017.

[26] A. Lendasse, V. Wertz, and M. Verleysen, "Model selection with cross-validations and bootstrapsapplication to time series prediction with RBFN models," Artificial Neural Networks and Neural Information Processing-ICANN/ICONIP 2003, pp. 174-174, 2003.

\section{AUTHORS}

Morteza Mohammadzaheri received his $\mathrm{PhD}$ from School of Mechanical Engineering, University of Adelaide, Australia in 2011. He has published/presented more than 90 peer-reviewed articles in technical journals and conferences. He is now an Assistant Professor of Dynamic Systems and Control at the Department of Mechanical and Industrial Engineering of Sultan Qaboos University, Oman.

Mojtaba Ghodsi received his B.Sc. degree in Mechanical Engineering from Isfahan University of Technology in 1999, the M.Sc. degree in Applied Mechanics from Tehran Polytechnic in 2001 and continued his research as Ph.D. (2007) and JSPS postdoctoral fellow (2009) in Precision Engineering Department of the University of Tokyo, Japan. Currently, he is pursuing his career at Sultan Qaboos University, Oman in department of Mechanical and Industrial Engineering. His main research interests include Smart Materials for Actuators, Sensors and Energy Harvesting, NDT and development of Mechatronic systems and devices. Dr. Ghodsi is a Member of IEEE and International Society of Optics and Photonics (SPIE).

Abdullah AlQallaf is an Assistant Professor with the Department of Electrical Engineering, Kuwait University. He received his Ph.D. degree in Electrical Engineering from the University of Minnesota-twin cities, St. Paul, MN, in 2009. Alqallaf's research interests are Microwave Imaging Techniques, Multimedia Signal Processing, Communication, Bioinformatics and Medical Image Analysis. Alqallaf is an IEEE Senior Member and an IEEE Board Member-Educational and Professional Activities-Kuwait section.
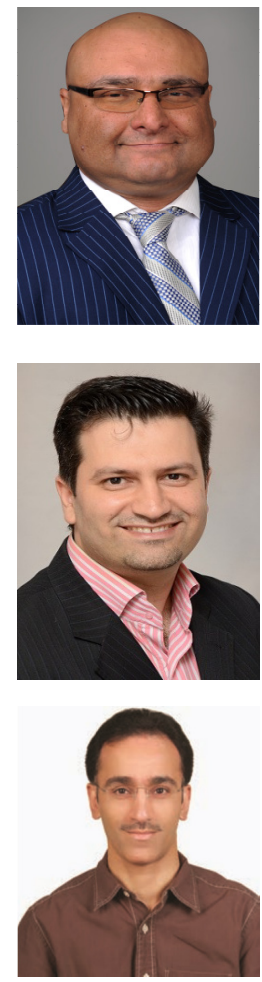